\title{
Posibles actuaciones psicológicas para reducir la conducción bajo los efectos del alcohol y viabilidad de su aplicación en España.
}

\author{
Maa Teresa Cortés Tomás*; José Antonio Giménez Costa** \\ * Departamento de Psicología Básica. Facultad de Psicología. Universidad de Valencia. Profesora Titular de Universidad. \\ **UPC (Unidad de Prevención Comunitaria de la Mancomunidad de la Canal de Navarrés). Psicólogo. \\ Enviar correspondencia a: \\ María Teresa Cortés Tomás. Departamento de Psicología Básica. Facultad de Psicología. Avda. Blasco Ibáñez, 21. 46010 Valencia. \\ Tfno: 963864420 (ext. 52280). E-mail: Maria.T.Cortes@uv.es. \\ Recibido: 14 de octubre de 2002. \\ Aceptado:: 8 de julio de 2003.
}

\section{RESUMEN}

Las recientes modificaciones en la legislación sobre Tráfico y Seguridad Vial (Ley 19/2001) reflejan la necesidad de clarificar los posibles procedimientos a realizar con los conductores sancionados por conducir bajo la influencia del alcohol. Tras verificar la reducida efectividad de las medidas aplicadas en España y revisar las actuaciones realizadas en otros países, destacamos los programas educativos y terapéuticos por ser las intervenciones más estructuradas y con resultados más favorables.

En este trabajo, presentamos una descripción de estos programas así como una serie de recomendaciones sobre la mejor manera de insertarlos en nuestra legislación. Entre las directrices que probablemente contribuirán a mejorar la efectividad de estas intervenciones destacamos, por ejemplo, su aplicación inmediata tras la infracción cometida, una vez agrupados los participantes en función de su patrón de consumo y de su estilo de vida. Esto nos lleva a proponer los programas educativos, más centrados en la conducción, para aquellos infractores con menor problemática de consumo y los programas terapéuticos para los infractores dependientes. Si bien, insistimos en la necesidad de no disponer de programas cerrados sino de adaptar la intervención a las necesidades de los asistentes.

Asimismo, destacamos la importancia de contar con monitores especializados en temas de tráfico y con conocimientos básicos sobre adicciones como responsables de los programas educativos, y con profesionales especializados en adicciones que trabajen en coordinación con los centros asistenciales convencionales, de modo que el infractor reciba un tratamiento bio-psico-social junto con el programa terapéutico. En ningún caso se considera que el programa terapéutico sea suficiente para evitar la reincidencia.

Palabras clave: Alcohol, Seguridad Vial, Intervención Psicología, Infractores, Legislación

\section{ABSTRACT}

Recent modifications to the legislation on Traffic and Road Safety (Act 19/2001) reflect the necessity of clarifying the possible procedures to be followed when dealing with drivers penalised for driving under the influence of alcohol. After verifying the limited efficacy of the measures applied in Spain, and after reviewing actions in other countries, we highlight educational and therapeutic programmes as being the most structured and having the most favourable results.

In this work, we present a description of these programmes as well as a number of recommendations on the best way of inserting them in our legislation. Among the guidelines that would probably contribute to improving the efficacy of these interventions, we highlight, for example, their immediate application following an infringement, once the participants are grouped in accordance with their consumption pattern and lifestyle. This leads us to propose educational programmes, based more on safe driving, for those offenders with minor consumption problems, and therapeutic programmes for alcohol-dependent offenders. Even so, we stress the need for not being restricted to closed programmes but to adjust interventions in accordance with the needs of those concerned.

In addition, we stress the importance of having instructors specialising in road safety matters and with basic knowledge of addictions as the ones responsible for the educational programmes, and with specialized professionals in addiction to coordinate work with the conventional treatment centres, in such a way that the offender receives a bio-psycho-social treatment together with the therapeutic programme. In no case is the therapeutic programme considered to be sufficient, on its own, in preventing a repeat of the offence.

Key words: Alcohol, Road Safety, Psychological Intervention, Traffic Offenders, Legislation 


\section{INTRODUCCIÓN}

$\mathbf{L}$ a conducción bajo los efectos del alcohol, sin necesidad de efectuarse bajo un estado de embriaguez, es uno de los grandes problemas que aparecen en el área de la Seguridad Vial (Cortés y Montoro, 2000; Sánchez, 1995).

Son múltiples las fuentes, tanto oficiales (Plan Nacional sobre Drogas -PNSD-, Instituto Nacional de Toxi- cología, Dirección General de Tráfico, etc.) como no oficiales (estudios en urgencias de hospitales, tesis doctorales, notas de prensa, etc.) que nos ofrecen información al respecto. En la Tabla 1 se resumen los últimos datos publicados por el Observatorio Español sobre Drogas -informe $n^{\circ} 5$, julio 2002- así como una comparación sobre la incidencia de esta problemática entre 1991/2000 citada en Álvarez y Del Río (2001).

Tabla 1. Datos epidemiológicos sobre la presencia de alcohol en conductores fallecidos en accidentes de tráfico .

\begin{tabular}{|c|c|c|c|c|}
\hline \multicolumn{2}{|c|}{ Instituto Nacional de Toxicología (1991-2000) } & \multicolumn{3}{|c|}{ Observatorio Español sobre Drogas (Informe $n^{\circ} 5$ ) } \\
\hline \multicolumn{2}{|c|}{ Muestra: 5745 conductores fallecidos } & \multicolumn{3}{|c|}{ Muestra: 1363 conductores fallecidos } \\
\hline \multicolumn{2}{|l|}{ Presencia de alcohol: 43'8\% } & \multicolumn{3}{|c|}{ Presencia de alcohol: $41,2 \%$} \\
\hline Descenso en casos detectados & $10^{\prime} 1 \%$ & \multicolumn{2}{|c|}{ Aumento en casos detectados (1990-2000) } & $4,3 \%$ \\
\hline $\begin{array}{l}\text { Descenso en el porcentaje de casos } \\
\text { superiores al límite legal }\end{array}$ & $14^{\prime} 2 \%$ & \multicolumn{2}{|c|}{$\begin{array}{l}\text { Casos con un nivel superior al límite legal } \\
\text { permitido del total de la muestra (2000) }\end{array}$} & $322^{\prime} 6 \%$ \\
\hline $\begin{array}{l}\text { Casos con un nivel superior al límite } \\
\text { legal permitido del total de la muestra }\end{array}$ & $35^{\prime} 8 \%$ & & & \\
\hline \multicolumn{5}{|c|}{ Casos con un nivel superior al límite legal permitido } \\
\hline $1998 \rightarrow 30^{\prime} 37 \%$ & \multicolumn{2}{|c|}{$1999 \rightarrow 30^{\prime} 06 \%$} & $2000 \rightarrow 32{ }^{\prime} 6 \%$ & \\
\hline
\end{tabular}

Más de un tercio de los conductores fallecidos en un accidente de tráfico muestran cantidades de alcohol positivas, una gran mayoría de los cuales -30 '37\%, $300^{\prime} 06 \%$ y 32 ' $6 \%$, lo que equivale aproximadamente a un $80 \%$ del total de casos con presencia de alcoholsuperan el nivel de alcoholemia legalmente permitido.

Sin embargo, la repercusión del consumo de alcohol no podemos conocerla plenamente a través de estos datos, pues va mucho más allá. Son múltiples los conductores que asumen un riesgo elevado, de manera repetida en muchas ocasiones, pero que no llegan a causar un accidente mortal, por lo que no aparecen en estas estadísticas. Para cubrir parte de esta limitación podemos recurrir a los datos sobre diligencias (vía penal) y denuncias (vía administrativa) por conducción con tasas de alcoholemia superiores al límite legal, ofrecidos también por el PNSD. A nivel general, el número de diligencias abiertas, tanto en los controles de alcoholemia como en las infracciones de tráfico y la negación a realizar las pruebas de alcoholemia, han disminuido ligeramente en el periodo desde 1998 a 2000. Sin embargo, en este mismo periodo, el número de denuncias practicadas ha experimentado un alarmante incremento (Tabla 2).

Tabla 2. Evolución de las diligencias (vía penal) y denuncias (vía administrativa) por conducir con una tasa de alcoholemia superior al límite legal.

\begin{tabular}{|lc|c|c|}
\cline { 2 - 4 } \multicolumn{1}{c|}{} & \multicolumn{1}{c}{1998} & 1999 & $\mathbf{2 0 0 0}$ \\
\hline Diligencia por accidente & 5.826 & 4.892 & 5.119 \\
\hline Diligencia por infracción & 5.731 & 3.953 & 3.753 \\
\hline Diligencia por negarse al control & 636 & 480 & 437 \\
\hline Diligencia por control & 22.582 & 18.679 & 15.934 \\
\hline Denuncias & 45.231 & 50.235 & 63.193 \\
\hline
\end{tabular}


En general, se produce una infraestimación de la presencia real del alcohol en nuestras carreteras, debido principalmente a que las fuentes de consulta suelen ofrecer los resultados de muestras concretas de la población de conductores infractores. Nos falta la visión de conjunto de cuál es la repercusión total de la presencia del alcohol.

Además, los datos extraídos presentan ciertos sesgos que es necesario tener en consideración. Por ejemplo, los controles de alcoholemia suelen realizarse de manera poco regular al estar sujetos a cuestiones de índole político-económicas; también es importante considerar que los datos detectados por sintomatología o infracción van a estar íntimamente ligados al nivel de formación en cuestiones de alcohol de los agentes de seguridad del Estado. Así mismo, en los datos aportados por accidentes -los más numerosos-, no están representados los casos de los que no se da parte a los agentes.

Otras variables relevantes a considerar hacen referencia directamente al consumo de alcohol. En este caso, no hay que olvidar el porcentaje de conductores con una tolerancia al alcohol que pueden pasar desapercibidos por no presentar una sintomatología manifiesta, o los accidentes que pueden estar causa- dos por un peatón consumidor. Esto último pone de manifiesto que en las estadísticas se obvia el papel de otros usuarios de la vía.

Pero a pesar de no tener un índice general exacto del número de infractores que conducen bajo los efectos del alcohol, el porcentaje estimado suele ser elevado. En el caso concreto de muertes por accidente de tráfico, casi la mitad de éstas son atribuidas al alcohol ( $n=2316 ; 40 \%$; Defunciones según la causa de muerte, 1997. INE 2000). Todo esto justifica la relevancia a la hora de plantear la necesidad de una intervención rigurosa que palie este problema.

Entre los pocos estudios que permiten planificar esta intervención encontramos las encuestas paneuropeas Social Attitudes to Road Traffic Risk in Europe -SARTRE 1 recogido en TABULA V (1992) y SARTRE 2, recogido en DGT (1998)- en las cuales se analizan tanto el comportamiento del conductor como los determinantes de su actitud: percepción del alcohol como factor de riesgo; conocimiento del límite legal y cómo se llega a éste; aceptación de medidas disuasorias y valoración personal de asociar el consumo de alcohol y la conducción. Las principales conclusiones extraídas en la encuesta de 1998 se enuncian en la Tabla 3.

Tabla 3. Principales conclusiones extraídas del informe SARTRE 2 (DGT,1998).

\section{CONCLUSIONES SARTRE, 1998}

1. El patrón de consumo de alcohol de los conductores españoles es el de consumidores frecuentes, pero moderados.

2. Estos asocian el alcohol-conducción en mayor proporción que en otros países.

3. No tienen una idea clara de los efectos de diferentes niveles de impregnación y confían demasiado en la experiencia en la conducción. Especialmente los varones de mediana edad se consideran inmunes a los efectos de la bebida.

4. Consideran que la probabilidad de ser sancionado y controlado por conducir bajo los efectos del alcohol es muy escasa.

5. No saben verificar cuando la cantidad de alcohol ingerida supera el nivel legal permitido.

6. Desconocen que conducir bajo los efectos del alcohol es conducir bajo los efectos de cualquier cantidad de alcohol, pues ya desde pequeñas cantidades se provocan cambios psicofísicos; que al ir incrementando la impregnación alcohólica, los efectos se multiplican. No es preciso llegar a estar "borracho" (si se entiende por ello perder todo el control sobre los propios actos) para estar en malas condiciones para conducir.

7. Hay que trabajar con grupos específicos que se muestran significativamente peor informados, pues para que el mensaje sea eficaz, tiene que ser lo más adaptado posible a los receptores sobre quienes se quiere actuar. No hay que olvidar que hay una cantidad importante de conductores que no beben.

8. Es preciso seguir promoviendo la información y sensibilización, así como aumentar el control real para que la percepción sobre la probabilidad de ser controlado también aumente y afecte a las decisiones que tomen los conductores.

Estas conclusiones nos ofrecen información importante sobre algunos de los contenidos que debemos tener en cuenta a la hora de diseñar posibles estrategias de intervención -(p. ej. el nivel de desconocimiento de la tasa de alcoholemia que presenta el propio sujeto o de las leyes vigentes, etc.)-, así como de la relevancia que tiene en el diseño de estas intervenciones el considerar el nivel de consumo de alcohol entre los conductores. Esto último exige intervenciones ajustadas a cada uno de estos niveles, pues las variables que inciden sobre el problema son diferentes. 
Es obvia la necesidad de realizar investigaciones que profundicen en estos perfiles pues esto nos permitirá conocer de manera más ajustada los verdaderos factores que intervienen en estas infracciones, pudiendo diseñar estrategias de actuación adecuadas para poder paliarlos. Estos estudios son importantes sobre todo en estos momentos en los que se nos plantea en la ley una posible reeducación de estos conductores.

Bajo esta perspectiva veamos inicialmente cuál es la respuesta actual que se da en nuestro país a los conductores sancionados por conducir bajo los efectos del alcohol. Tras valorar la adecuación de estas medidas se presentarán algunas actuaciones aplicadas en otros países, finalizando con una posible combinación de todas estas medidas ajustadas a la situación española.

\section{RESPUESTA ESPAÑOLA ANTE LA CONDUCCIÓN BAJO LOS EFECTOS DEL ALCOHOL: MEDIDAS LEGISLATIVAS.}

Hasta el momento, en España la conducción por encima de los límites legalmente permitidos, así como cualquier conducción arriesgada a consecuencia de un consumo de alcohol por parte del conductor, recibe una sanción legal que en la mayor parte de las ocasiones es de carácter administrativo, limitándose a una multa económica y a la retirada del permiso o licencia de conducción.

En los últimos años se ha producido un endurecimiento tanto de las medidas legales adoptadas, como de las sanciones impuestas (Tabla 4). Destaca por ejemplo, la reducción de las tasas de alcohol permitidas entre los conductores (RD 2282/1998), que entró en vigor en mayo de 1999, así como la consideración de esta conducta como una infracción muy grave.

Entre los cambios legislativos encontramos otro tipo de medidas que inciden sobre el consumo, mostrando un efecto indirecto sobre la conducción. Por ejemplo, en nuestra Comunidad aparecen reflejados en el Decreto Legislativo 1/2003 de 1 de abril, aspectos como la edad mínima para consumir alcohol y para poder acceder y disponer de esta sustancia.

Sin embargo, la investigación ha demostrado que las medidas legales, cuando se aplican de modo aislado, no resultan todo lo efectivas que se pretende, de

Tabla 4. Principales normas en vigor en España diferenciadas por actuación legal.

\begin{tabular}{|c|c|}
\hline MEDIDAS & LEYES ESPAÑOLAS \\
\hline $\begin{array}{l}\text { Límite máximo de. } \\
\text { alcohol permitido }\end{array}$ & $\begin{array}{l}\text { - RD 2282/1998. Reglamento General de Vehículos. } \\
\text { Establece el límite máximo permitido de alcohol en sangre } \\
\text { para conductores de turismos en } 0^{\prime} 5 \mathrm{~g} / \mathrm{l} \text {; para transportes } \\
\text { especiales y "novatos" en } 0,3 \mathrm{~g} / \mathrm{l} \text { de alcohol en sangre. }\end{array}$ \\
\hline Sanciones: & $\begin{array}{l}\text { - Ley 19/2001 Reforma de la Ley sobre Tráfico, Circulación } \\
\text { de vehículos a motor y Seguridad Vial (RD 339/1990) }\end{array}$ \\
\hline \multicolumn{2}{|l|}{ - Económicas } \\
\hline \multirow[t]{7}{*}{ - Sobre la privación de libertad. } & $\begin{array}{l}\text { Son infracciones Muy Graves, cuando no sean constitutivas de } \\
\text { delito: }\end{array}$ \\
\hline & $\begin{array}{l}\text { - La conducción por las vías habiendo ingerido bebidas alcohólicas } \\
\text { con tasas superiores a las reglamentariamente permitidas. }\end{array}$ \\
\hline & $\begin{array}{l}\text { - Incumplir con la obligación de someterse a las pruebas que se } \\
\text { establezcan para la detección de posibles intoxicaciones de } \\
\text { alcohol. }\end{array}$ \\
\hline & $\begin{array}{l}\text { Estas infracciones serán sancionadas con multa de } 302 € \text { a } 602 € \\
\text { y suspensión del permiso o licencia hasta } 3 \text { meses. }\end{array}$ \\
\hline & - Ley Orgánica 10/1995: \\
\hline & $\begin{array}{l}\text { - Conducir bajo los efectos del alcohol: arresto de } 8 \text { a } 12 \text { fines de } \\
\text { semana o multa de } 3 \text { a } 8 \text { meses. Privación permiso de conducir } \\
\text { entre } 1 \text { y } 4 \text { años. }\end{array}$ \\
\hline & $\begin{array}{l}\text { - Negarse a hacer la prueba de alcoholemia: delito de desobe- } \\
\text { diencia grave (art. 556): prisión de } 6 \text { meses a } 1 \text { año. }\end{array}$ \\
\hline
\end{tabular}


cara a reducir la frecuencia con que se asocia el consumo de alcohol y la conducción (Hedlung, 1995; Homel, 1994; Martín, Annan y Forst, 1993; Peck, 1991; WeIls-Parker y Cosby, 1988). Tal como hemos expuesto en otros trabajos (Cortés y Montoro, 2000), entre las razones que pueden explicar la reducida efectividad de las mismas destacan:

- la baja expectativa que tienen los sujetos de ser detenidos. Aunque tras la puesta en marcha de una nueva medida restrictiva, los conductores reducen su conducta por miedo a ser sancionados, cuando se dan cuenta de que la probabilidad de que esto suceda es muy baja, disminuyen sus expectativas de sanción y vuelven a asociar ambas conductas. Sería necesario elevar el riesgo real de arresto o sanción, manteniéndolo a este nivel durante mucho tiempo.

- el poco control que se tiene de las reincidencias y del cumplimiento de las sanciones. Algunas investigaciones defienden que casi un $75 \%$ de los sujetos continúan conduciendo aunque se les haya retirado la licencia de conducción (Marowitz y DeYoung, 1995; Sadler y Perrine, 1984; Voas, Scott y Lange, 1997; Yu y Willford, 1995). Este poco control está propiciando que una conducta anómala no sea castigada, al tiempo que se refuerza la continuidad de la misma.

- la forma de aplicar la sanción. Sabemos, por la investigación psicológica, que un castigo resulta efectivo, entre otras cosas, cuando se aplica inmediatamente tras la conducta que quiere reducirse (la conducción bajo los efectos del alcohol) y además de forma reiterada (sancionar cada vez que el sujeto realice la conducta). En los casos de conducción bajo los efectos del alcohol, no se cumple ninguna de estas dos condiciones, pues la sanción la recibe el sujeto meses después de haber cometido la infracción, por lo que no puede asociar su mala actuación con la sanción impuesta, y su conducta no es sancionada cada vez que la realiza. Esto hace que el sujeto no tome conciencia de que está haciendo algo inadecuado por lo que no se consigue el objetivo final pretendido: reducir esta conducta en el sujeto. En estos momentos con la Ley de Juicios Rápidos (Ley Orgánica 8/2002 y Ley 38/2002) se intenta contrarrestar la inadecuación en la aplicación del castigo al menos en aquellos delitos contra la seguridad del tráfico que requieren de instrucción sencilla.

- el desconocimiento del propio patrón de consumo. Albery y Guppy (1995) aluden a la reducida habilidad que muestran los sujetos para identificar cuando su consumo ha alcanzado el límite legalmente establecido. Es frecuente que estos conductores se consideren capacitados para conducir con seguridad, cuando su tasa de alcoholemia ha superado con creces lo permitido legalmente. Además, este problema se acentúa conforme los sujetos realizan consumos de alcohol más elevados. En estos casos, aparecen percepciones mucho más deterioradas sobre las posibles consecuencias que pueden derivarse del consumo realizado.

Considerando que este desconocimiento existe actualmente entre los conductores españoles (ver Tabla 3), es obvio que no puede solucionarse el problema de la conducción bajo los efectos del alcohol, únicamente con medidas de carácter sancionador, pues con ello no logramos que el sujeto aprenda a determinar cuando su nivel de alcoholemia está llegando a los límites legales, ni tampoco le ayudamos a que consiga modificar su percepción errónea sobre los efectos que el consumo de alcohol puede tener en su organismo y capacidades.

- la dependencia al alcohol. Si el sujeto es dependiente del alcohol, las sanciones, por severas que sean, suelen tener muy poco efecto (Hedlung, 1995; Wells-Parker, 1995). En estos casos hay que considerar la solución a la pérdida de control y a otros factores asociados con ese consumo desviado.

Estas y otras razones -la idea de que no se está incidiendo sobre los factores que podríamos llamar nucleares (el consumo del sujeto y la seguridad en su habilidad de conducción), así como el que los resultados obtenidos con las medidas represivas sean limitados en el tiempo (Roos, 1975; Reed, 1982)-, nos llevan a plantear la necesidad de proponer otro tipo de intervenciones que complementen las de carácter legal.

Aceptamos la recomendación de Bartl, Assailly, Chatenet, Hatakka, Keskinen y Willmes-Lelz, (2002); Comisión de las Comunidades Europeas (2001); Marowitz y DeYoung (1995); National Transportation Safety Board (NTBS) (1984); Nichols y Ross (1990); Presidential Commision on Drunk Driving (1983); Preusser, Blomberg y Ulmer (1988), y Transportation Research Board (1995), de combinar las medidas legislativas (actualmente vigentes en España) con la educación y/ o tratamiento sobre alcohol, como la mejor estrategia para combatir la conducción bajo los efectos del alcohol, combinación esta que se ha mostrado efectiva en otros países para conseguir resultados favorables, tanto a corto como a largo plazo. Los estudios europeos recogidos en el proyecto ANDREA (Bartl et al., 2002) indican que la tasa de reincidencia de los participantes en estos cursos es aproximadamente la mitad de la que presentan los sujetos sancionados que no asisten a este tipo de programas. 
PROPUESTAS COMPLEMENTARIAS A LAS MEDIDAS LEGISLATIVAS: PROGRAMAS EDUCATIVOS Y TERAPÉUTICOS.

Tras la revisión exhaustiva que hemos realizado de las actuaciones aplicadas en otros países entre los conductores sancionados por conducir bajo la influencia del alcohol (Cortés y Montoro, 2000; Giménez y Cortés, 2000; INTRAS, 2000) Ilegamos a la conclusión, confirmada posteriormente por otros investigadores (Bartl et al., 2002), de que ante la diversidad de actuaciones que se plantean, destacan los programas educativos y terapéuticos por su abordaje del problema desde un mayor número de áreas interrelacionadas. Los primeros se aplican desde los años sesenta, mientras que los terapéuticos lo hacen desde los setenta, lo que ha permitido ir concretando a lo largo del tiempo tanto sus objetivos como las ventajas e inconvenientes de unos y otros.

Algunos objetivos, señalados con un punto, (Tabla 5) son compartidos por ambos tipos de programas ( $p$. ej. el incremento en el nivel de conocimiento o la toma de conciencia de su propia forma de consumir alcohol), mientras que otros (señalados con una flecha o un cuadrado) son específicos de cada uno de ellos. En líneas generales, podemos afirmar que los programas educativos se dirigen a los conductores sin problemas con el consumo de alcohol, mientras que los terapéuticos parecen más adecuados para sujetos que muestran un abuso o una dependencia alcohólica.

Tabla 5. Objetivo general y específicos de los principales tipos de intervención realizados en otros países con conductores sancionados por conducir bajo los efectos del alcohol.

\begin{tabular}{|c|c|c|}
\hline & OBJETIVO GENERAL & OBJETIVOS ESPECÍFICOS \\
\hline $\begin{array}{l}\text { PROGRAMAS } \\
\text { EDUCATIVOS }\end{array}$ & $\begin{array}{l}\text { Pretenden conseguir, } \\
\text { ofreciendo información, } \\
\text { un cambio de actitud que } \\
\text { se traduzca finalmente en } \\
\text { un cambio de conducta }\end{array}$ & $\begin{array}{l}\text { - Incremento del nivel de conocimiento del } \\
\text { sujeto (legislación sobre alcohol-conducción, } \\
\text { efectos del consumo de alcohol sobre la } \\
\text { habilidad de conducir...). } \\
\Leftrightarrow \text { Toma de conciencia de su propio consumo. } \\
\Leftrightarrow \text { Análisis de la infracción cometida. } \\
\text { - Determinación de los antecedentes de la } \\
\text { conducta. } \\
\text { - Cambio de actitud. } \\
\Leftrightarrow \text { Cambio en su estilo de vida. } \\
\Leftrightarrow \text { Disminución de la reincidencia. }\end{array}$ \\
\hline $\begin{array}{l}\text { PROGRAMAS } \\
\text { TERAPÉUTICOS }\end{array}$ & $\begin{array}{l}\text { Intentan modificar } \\
\text { los patrones de consumo } \\
\text { de alcohol de los } \\
\text { participantes }\end{array}$ & $\begin{array}{l}\text { Cambio en la conducta de consumo de alcohol. } \\
\text { Identificación de posibles factores de riesgo. } \\
\text { Desarrollo o adquisición de recursos de } \\
\text { afrontamiento de carácter cognitivo o } \\
\text { conductual. } \\
\text { Derivación a otros recursos. } \\
\text { - Cambio de actitud. } \\
\text { - Incremento del nivel de conocimiento. } \\
\text { - Toma de conciencia de su propio consumo. }\end{array}$ \\
\hline
\end{tabular}

Del mismo modo que muestran similitudes en algunos de sus contenidos, también lo hacen en cuestiones de carácter más formal y metodológico. En ambos casos, se forman grupos heterogéneos de conductores, de entre 8 y 12 asistentes, que son remitidos por el juzgado, estando obligados a cumplir con el programa para poder recuperar su licencia de conducción. A estos se les exige una participación activa de modo que sean ellos mismos los que planteen las necesidades específicas que poseen, así como las posibles alternativas a las mismas. De este modo, cada programa va a tener sus propias peculiaridades dependiendo de las características de los participantes.
Conforme el programa se hace más clínico (terapéutico) se incrementa el tiempo de aplicación del mismo, lo que es justificable por el tipo de población al que va dirigido. Ya hemos enunciado anteriormente que estos programas se realizan con consumidores que pueden calificarse de abusadores o dependientes del alcohol, por lo que se requiere una intervención inicial específica en temas de alcohol, completada posteriormente con contenidos propios de alcohol-conducción. En estos casos, debemos analizar con mayor detalle aspectos tales como los determinantes de la conducta o el modo de afrontarlos. Todo esto requiere de más tiempo para poder tratarse minuciosamente. 
No perdamos de vista que estos sujetos han establecido un hábito asociando reiteradas veces el consumo de alcohol con la conducción, y un hábito requiere de más tiempo para poder ser modificado y para que esta modificación perdure en el tiempo.

A grandes rasgos, estaríamos hablando de un continuo de intervención, en el que destacarían actuaciones de carácter educativo en el polo inferior -dirigidas a sujetos con pocos problemas de consumo- e intervenciones de carácter terapéutico en el polo superior, orientadas a sujetos con un abuso o una dependencia al alcohol. En ningún caso, la meta de los programas educativos y de las intervenciones complementarias a un tratamiento de alcoholismo es que el sujeto se mantenga abstinente, sino que no asocie la conducción una vez ha bebido alcohol (Cortés y Montoro, 2000), aunque es lógico pensar que éste va a ser un objetivo fundamental en la mayoría de los programas asistenciales que siga la población dependiente.

Estas intervenciones, tanto educativas como terapéuticas, aplicadas ya en otros países, han mostrado tener una serie de ventajas, pero también toda una serie de inconvenientes que pasamos a detallar seguidamente.

Entre las ventajas de las actuaciones educativas, destacar el incremento en el nivel de conocimiento (Bartl et al., 2002; Brown, 1980; Davidson, 1983 -citado en Liban; Vingilis y Blefgen, 1987-; Kooler y Bruvold, 1992; Vingilis, Chung y Adlaf, 1981), así como los cambios de actitud ante la conducción bajo los efectos del alcohol (Brown, 1980; Davidson, 1983 -citado en Liban et al., 1987-; Eddy, 1976; Kooler y Bruvold, 1992; Vingilis, Chung y Adlaf, 1981), una reducción de la reincidencia en este tipo de infracciones (Bartl et al., 2002; Kooler y Bruvold, 1992; McGuire, 1982; Nichols, Weinstein, Ellingstad y Struckman-Johnson, 1978; Reis, 1980) y un juicio más realista sobre su capacidad para conducir tras el consumo (Edelman; Walker; Morris y Godfrey, 1977). En algunos casos, se ha conseguido indirectamente un objetivo no perseguido (Brown, 1980), el descenso en los niveles de consumo.

En cuanto a los programas terapéuticos resulta complicado hacer afirmaciones tajantes sobre los resultados obtenidos, ya que existe una gran variabilidad en los mismos, a pesar de ser menor el número de programas aplicados. En líneas generales, podemos concluir que se da un descenso generalizado del consumo de alcohol previo a la conducción (Haguen, Williams y McConnell, 1979), un aumento de la responsabilidad del conductor para evitar accidentes (Spoerer, Ruby y Jennsch, 1997), juicios más realistas sobre su propia capacidad para conducir tras haber consumido (Spoerer, Ruby y Jennsch, 1997), un incremento significativo del conocimiento (Bartl et al., 2002; Edelman et al., 1977) y una reducción de las infracciones relacionadas con el consumo de alcohol (Bartl et al., 2002; Seixas y Hopson, 1975).

Ambos tipos de actuaciones comparten la mayoría de inconvenientes o limitaciones. Una de las más importantes hace referencia al tipo de población sobre la que se interviene, pues suele ser muy heterogénea tanto en el consumo como en el estilo de vida (Bartl et al., 2002; Cortés y Montoro, 2000; Giménez y Cortés, 2000). Esto lleva a que se obtengan resultados contradictorios dentro de una misma actuación, siendo ésta más adecuada para unos sujetos que para otros. Sería conveniente establecer grupos homogéneos de participantes, ajustando al máximo la intervención a la población concreta a la que va dirigida. Esto podría llevarse a cabo, por ejemplo, derivando a los sujetos a estos programas, no directamente desde los juzgados, sino a partir de la evaluación realizada por un equipo de profesionales encargados de organizar a los posibles participantes en grupos.

Otra de las limitaciones que debemos tener en cuenta es que los sujetos malinterpreten que lo que se les está enseñando en el programa es cómo beber y conducir sin ser descubierto por las Fuerzas de Seguridad del Estado. Este problema podría solucionarse formando a los terapeutas o responsables que van a impartir estos programas para que eviten las posturas ambivalentes.

Una limitación específica de los programas educativos es la creencia errónea que subyace en su base respecto a que la información conlleva necesariamente un cambio actitudinal y por consiguiente un cambio de conducta. Una posible solución en este sentido sería adoptar un modelo teórico de base que mostrase cuáles son las variables que realmente pueden estar afectando a la actitud del sujeto y a su conducta, y que por lo tanto nos indicase aquellas áreas sobre las que deberíamos centrar nuestra intervención.

Finalmente mencionar algunas limitaciones metodológicas encontradas en la evaluación de estos programas. Entre estas destacan por ejemplo errores en el diseño experimental (p.ej. la utilización de grupos de control inadecuados, la inexistencia de estos, o los diseños con muestras muy pequeñas), solucionables todos ellos mediante una adecuada selección de los grupos control y experimental; o la debilidad que muestran algunas de las escalas de evaluación, por lo que sería conveniente utilizar pruebas validadas o estudiar la posibilidad de desarrollar alguna escala nueva que se ajustase a las necesidades específicas de evaluación de cada intervención. Por último, destaca el que no haya una unificación en la duración de los períodos de seguimiento, lo que dificulta conocer si se mantienen los resultados obtenidos con el paso del tiempo, o poder comparar los resultados de diferentes intervenciones. En nuestro caso, esto podría solucionarse estableciendo un periodo de seguimiento están- 
dar, que si atendemos a la ley vigente (Artículo 82, Ley 19/2001) podríamos situar en los 2 años.

Como hemos visto, el diseño de estos programas no es tarea sencilla, como tampoco lo es su aplicación. En primer lugar, se debe contar con un marco legal que ampare y regule este tipo de intervenciones, marco que en España se presenta favorable desde dos posibles vías: el Código Penal y La Ley de Tráfico y Seguridad Vial 19/2001.

\section{POSIBILIDAD DE APLICACIÓN DE ESTE TIPO DE PROGRAMAS EN ESPAÑA.}

Concretamente la aplicación de estos programas a aquellas personas condenadas a penas de prisión por la comisión de delitos contra la seguridad del tráfico, puede justificarse directamente en el Código Penal en función de los supuestos de suspensión de la condena (arts. 80 a 87 del CP).

Existe la posibilidad de cambiar la pena de prisión por la participación en programas de educación vial, con carácter obligatorio (art. 83.1.4). Las condiciones que deben cumplir los destinatarios de estos programas son tener satisfecha su responsabilidad civil, y que sea su primera condena (art. 81). Siendo así, los sujetos reincidentes quedan excluidos, punto a estudiar para su posible introducción en el marco legal, ya que en otros países (Whitehead et al., 1984 -citado en Liban, Vingilis y Blefgen, 1987-; Winkler, Jacobshaguen y Nickel, 1990; Spoerer, Ruby y Jennsch, 1997) se han diseñado programas para este tipo de población obteniéndose con ellos resultados muy positivos.

Además, la suspensión de la condena debe realizarse con la mayor brevedad posible (art. 82) y durante un periodo de entre 2 y 5 años (art. 80.2) que no es necesario agotar. Lo normal en este sentido es que los programas tengan una duración de mes y medio a dos meses, pudiéndose utilizar el resto del plazo legalmente establecido como un periodo de seguimiento del sujeto. Durante todo este periodo de suspensión, el sujeto debe cumplir con una serie de obligaciones, el incumplimiento de las cuales daría lugar a la revocación de la suspensión, y al cumplimiento de la condena suspendida (art. 84). En caso contrario, es decir, si el sujeto cumple con todas las obligaciones satisfactoriamente, se considera que su pena ha remitido (art. 85.2).

También se establecen en el Código Penal dos métodos de seguimiento del cumplimiento de estas obligaciones. Estos son la comparecencia del infractor periódicamente ante el Juez, y la remisión de informes al Juzgado por parte de la Administración. Ahora bien, en España no disponemos actualmente de ninguna figura administrativa que pueda cumplir con el papel de oficial de libertad condicional, a diferencia de otros países como Alemania que sí lo poseen.

Existen otras posibilidades, no citadas expresamente por el legislador, pero deducibles mediante la interpretación de los procesos penales, que permiten aplicar estos cursos: sustituyendo la pena privativa de libertad mediante la realización de trabajos en beneficio de la comunidad -art. 379 en relación con el art. 88.2 del CP- o como medida de seguridad, conforme al juego que ofrecen los art. 99.6 y 105.1 en relación con el art. 83.4 del CP. De ambas posibilidades interpretativas da cuenta el Fiscal Coordinador del Tribunal Superior de Justicia de la Comunidad Valenciana (ver Dolz, 1998).

Además, desde el año 2001 disponemos de otra posible vía de remisión, contemplada en la Reforma de la Ley sobre Tráfico, Circulación de Vehículos a Motor y Seguridad Vial. En ésta se permite sustituir, de manera voluntaria, hasta un $30 \%$ de la sanción pecuniaria y del período de suspensión, por medidas reeducadoras consistentes en cursos formativos de comportamiento en materia de seguridad vial o módulos de concienciación sobre las consecuencias de los accidentes de tráfico. En el caso de incumplir estas medidas reeducadoras, y por tanto no haber superado con aprovechamiento y dentro del plazo las condiciones reglamentariamente determinadas, la persona está obligada al pago inmediato de la multa sustituida junto con los recargos pertinentes.

En el caso de conductores reincidentes, además de la sanción pecuniaria correspondiente, se les revoca su permiso de conducción, a no ser que de manera voluntaria soliciten realizar un curso de reciclaje y sensibilización en un centro autorizado. En este caso, a la sanción económica se le sumaría una suspensión de la licencia o permiso de conducir, de hasta tres meses.

A pesar de que en la actualidad ya disponemos de un apoyo institucional para poder llevar a cabo estos cursos, todavía nos queda por concretar la institución o responsable de los mismos, el personal encargado de llevarlos a cabo, su duración e incluso el lugar de realización.

Si aunamos todo lo presentado hasta el momento en este artículo, podemos encontrar algunas respuestas a estas cuestiones.

\section{CONCLUSIONES}

Al plantearnos cualquier intervención sobre los sujetos sancionados por conducir bajo los efectos del alcohol, nos damos cuenta de la imposibilidad de planificar una única actuación homogénea para todos ellos, pues estamos ante una población diversa en cuanto al consumo de alcohol se refiere. 
Mann, Smart y Anglin (1996) defienden la existencia de subgrupos de población que presentan diferentes relaciones entre su consumo de alcohol y las incidencias en la conducción derivadas del mismo. Esta idea ha sido matizada por otros investigadores como Bartl et al. (2002) y Wells-Parker (1995) los cuales llegan a afirmar tajantemente que hay que tratar de forma diferente a los sujetos que conducen a menudo con altos niveles de alcohol en sangre y a los infractores que una noche pueden haber superado el límite legalmente permitido, sin ser conscientes de estar cometiendo una infracción.

El motivo de esta recomendación también se fundamenta en una limitación que presentan algunos de los programas que hemos revisado. Se ha visto que cuando se incluye una mezcla heterogénea de sujetos, no todos resultan adecuados intelectual, cognitiva o socialmente para recibir el mismo tipo de intervención, lo que lleva asociada una disminución de la efectividad de los programas. Por este motivo, parece razonable que la actuación más lógica y adecuada (Cortés y Montoro, 2000) sea la de estructurar distintas intervenciones en función del nivel de consumo y de las características de los sujetos, tanto relacionadas con la conducción (por ejemplo si es la primera vez que son sancionados por esta conducta o si son reincidentes) como con su propio estilo de vida (situaciones en las que asocian el consumo de alcohol con una conducción posterior).

No consideramos adecuado incluir en este tipo de programas a los sujetos que muestran una dependencia al alcohol, pues en este caso el problema de la conducción es secundario al de su consumo. Nuestra recomendación en este caso es la derivación a un servicio de tratamiento del alcoholismo, complementado con algunas sesiones específicas dedicadas a la conducta de la conducción y su relación con el consumo de alcohol.

Así pues, independientemente de la vía de remisión -penal o administrativa-, la primera tarea a realizar, una vez el sujeto ha sido sancionado y se ha comprobado su adecuación para formar parte de un programa de este tipo, es evaluarlo en los dos aspectos señalados.

Tal como aparece en el Gráfico 1, recomendamos que esta evaluación la realice personal especializado en el tema. Atendiendo al Reglamento General de Conductores, el diagnóstico debe ajustarse a las prescripciones de una adecuada evaluación de las aptitudes psicofísicas del sujeto y, como tal, ser realizado por un equipo médico-psicológico que determine, a través de un informe cualificado, su idoneidad para la intervención propuesta. Este mismo equipo sería el encargado de recoger información sobre algunas variables relacionadas con el estilo de vida.

Gráfico 1. Principales comentarios extraídos sobre la viabilidad de aplicar estas actuaciones en España, superando las limitaciones que presentan.

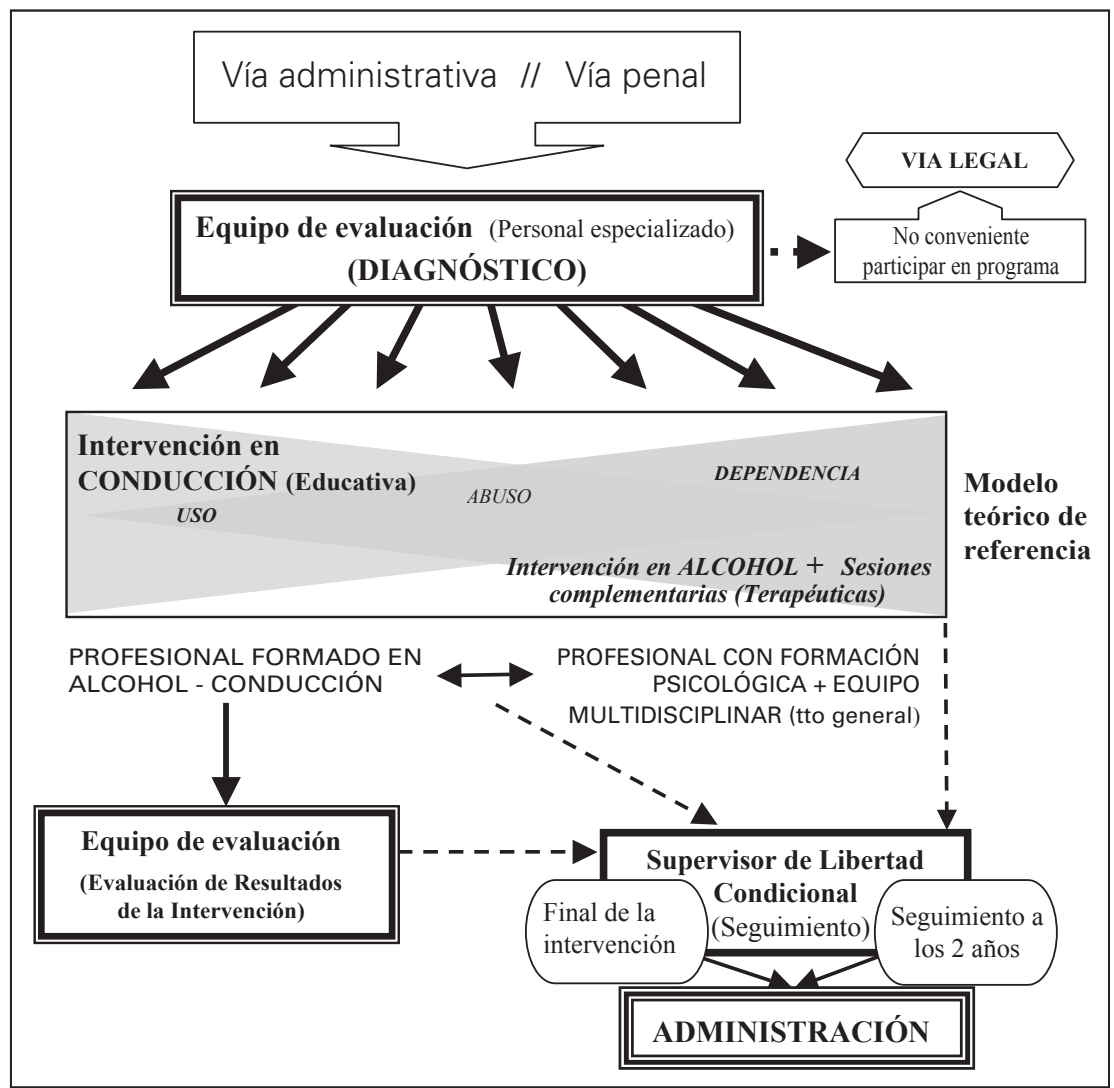


Investigadores en el tema como Malfetti (1975) ya indicaban que el número de sesiones, el contenido y los métodos utilizados en los programas fuesen seleccionados en función de las características y las necesidades de los infractores, aunque es cierto que apreciamos una estructura de base en todos los programas consultados.

Consideramos que el mejor programa posible debería tener un carácter modular, lo que significa que incluiría un amplio conjunto de actividades que podrían utilizarse dependiendo de las áreas deficitarias. Concretamente, tendríamos un conjunto de actividades comunes en todos los programas (presentación de objetivos; consecuencias del alcohol sobre la conducción; análisis de las infracciones...) y otro conjunto que, dependiendo de las características del grupo serían más o menos específicas (presión social para conducir bajo la influencia del alcohol; creencias y actitudes de los sujetos ante esta conducta...). Este tipo de cursos se utilizan sobre todo cuando se sabe poco de las características de los asistentes y ese es nuestro caso, pues cada grupo lo formarán personas que pueden presentar deficiencias muy heterogéneas.

En cuanto al método de trabajo más utilizado por su efectividad en los diferentes programas revisados destacan las técnicas grupales. Son múltiples las razones aludidas al respecto, por ejemplo el que facilitan a los sujetos disminuir los sentimientos de vergüenza, de infravaloración y de aislamiento que puedan surgirles; al tiempo que les permite verse a sí mismos reflejados en los demás mediante un proceso de identificación. Además, les permite observar cómo otras personas aplican en la vida real las técnicas trabajadas en las sesiones, facilitándoles su aprendizaje y la generalización del mismo.

Se propone por tanto, no una intervención educativa diferenciada de una terapéutica, como solemos encontrar en la literatura internacional, sino una intervención continua ajustada a las características más adecuadas de cada grupo de infractores (ver Gráfico 1). Lo que caracterizaría a los grupos situados en el nivel inferior sería una intervención más centrada en los aspectos de la conducción, mientras que en los grupos del otro extremo se combinaría la intervención convencional por consumo, con aspectos de la conducción (pues en este último caso el problema prioritario es el consumo de alcohol). En medio de estos dos extremos se situarían otros tipos de intervenciones que combinarían contenidos educativos con aquellos centrados en el control del consumo, según las necesidades de los sujetos.

En cuanto a los profesionales implicados en el desarrollo de estas intervenciones, es lógico que cuanta mayor repercusión tenga el consumo de alcohol mayor especialización en este tema se requerirá por su parte. Concretamente, continuamos defendiendo la necesidad de contar con profesionales con formación psicológica (Cortés y Montoro, 2000), consideración esta apoyada experimentalmente en las conclusiones del proyecto ANDREA (Bartl et al., 2002). En éste se recomienda explícitamente que los profesionales encargados de implementar el programa posean una formación psicológica que les permita manejar adecuadamente las dinámicas de grupo, motivar a los clientes más resistentes al cambio (autorreflexión), etc., pues se ha visto que esta formación es determinante para la obtención de mejores resultados. Incluso al comparar los objetivos alcanzados por estos profesionales con los conseguidos por técnicos formados únicamente en temas de alcohol, continúan siendo los primeros los que obtienen una mayor efectividad.

Puntualizar que en el caso de los programas terapéuticos -sesiones de alcohol-conducción adicionales a su tratamiento por alcoholismo- este profesional tendría que trabajar en coordinación con el equipo multidisciplinar encargado del tratamiento de la dependencia alcohólica del sujeto.

Además, no es conveniente que la evaluación de la intervención, la realicen los propios instructores del curso. En este sentido, se propone formar un equipo de evaluación encargado tanto de la agrupación inicial de los sujetos en función de sus características, como de la evaluación final del aprovechamiento.

En cuanto al seguimiento del cumplimiento por parte de los participantes, en el resto de países se recurre a la figura del Supervisor de Libertad Condicional, el cual recoge la información tanto de los participantes, como de los instructores del programa y del equipo de evaluación. Estos informes se envían a la administración correspondiente para que realicen el seguimiento del cumplimiento de las obligaciones de cada sujeto.

En estos momentos existen ya en España algunos intentos de intervención orientados hacia el establecimiento de programas educativo-terapéuticos (PAID -citado en Torres y Cortés, 1998-; PROSA -INTRAS, 2000-) que sin duda están abriendo las puertas hacia una intervención formalizada similar a la que desde hace tanto tiempo ya existe en otros países.

\section{REFERENCIAS}

Albery, I.P.; Guppy, A. (1995). Drivers' differential perceptions of legal and safe driving consumption. Addiction, 90, 245-254.

Alvárez, F.J. y Del Río, M.C. (2001). Alcohol y accidentes de tráfico. ¿Prevenir qué?. Trastornos Adictivos, 3, 172-180.

Bartl, G., Assailly, J.P., Chatenet, F., Hatakka, M., Keskinen, E. y Willmes-Lelz, G. (2002). EU Project "ANDREA": Analysis of driver rehabilitation programmes. En: Department of Transport, Behavioral Research in Road Safety: Twelfht Seminar (pp 36-45). London: Department of Transport. 
Brown, R.A. (1980). Conventional education and controlled drinking education courses witch convicted drunken drivers. Behavior Therapist, 11, 632-642.

Comisión de las Comunidades Europeas (2001). Libro blanco: la política europea de transportes de cara al 2010: la hora de la verdad. Bruselas: Comisión de las Comunidades Europeas.

Cortes, M.T. y Montoro, L. (2000, marzo). Primeras actuaciones de carácter rehabilitador en España ante una conducción bajo los efectos del alcohol. Comunicación presentada en las XXVII Jornadas Nacionales Socidrogalcohol, Madrid.

Dirección General de Tráfico (1998). SARTRE 2 Actitudes y comportamientos frente al riesgo vial de los conductores europeos (España comparada con el resto de los países). [Informe interno].

Dolz Lago, M.J. (1998). Los programas de educación vial ante los delitos contra la seguridad del tráfico: una alternativa eficaz. Actualidad penal, 1, 257-275.

Eddy, J. (1976). A DWI educational programme. Journal of Drug Education, 6, 137-139.

Edelman, S., Walker, D., Morris, P. y Godfrey, K. (1977). Impaired driving: A critical review of the research literature. Vancouver: Ministry of the Attorney-General.

Giménez, J.A. y Cortés, M.T. (2000, junio). La conducción bajo la influencia del alcohol: Estrategias de intervención. Comunicación presentada en las X Jornadas Socidrogalcohol de la Comunidad Valenciana, Alcoy.

Haguen, R.E., Williams, R.L. y McConnell, E.J. (1979). The traffic safety impact of alcohol abuse treatment as an alternative to mandated licensing controls. Accident, Analysis and Prevention, 11, 275-292.

Hedlung, J. (1995). Meta-analisys helps to define the policity-relevance of drink driving research. Addiction, 90, 1593-1594.

Homel, R. (1994). Drink-driving law enforcement and the legal blood alcohol limit in New South Wales. Accident, Analysis and Prevention, 26, 147-155.

INTRAS (2000). Elaboración de un programa piloto de intervención dirigido a sancionados por conducir bajo los efectos del alcohol (PROSA). [Informe Interno].

Kooler, J.M. y Bruvold, W.H. (1992). Evaluation of an educational intervention upon knoweledge, attitudes, and behavior concerning drinking/drugged driving. Journal of Drug Education, 22, 87-100.

Liban, C.B., Vingilis, E.R., y Blefgen, H. (1987). The canadian drinking-driving countermeasure experience. Accident, Analysis and Prevention, 19), 15-181.

Malfetti, J.L. (1975). Reeducation and rehabilitation of the drunken driver. Journal of Drug Issues, 5, 255-269.

Mann, R.E., Smart, R.G. y Anglin, L. (1996). Alcohol related measures as factors in traffic fatalities. Journal of Studies on Alcohol, 57, 646-651.

Marowitz, L. y DeYoung, D. (1995). Drunk-driving recidivism: predicting factors from arrest context and case disposition: Comment. Journal of Studies on alcohol, 57,679 .

Martin, S.E., Annan, S. y Forst, B. (1993). The special deterrent effects of a jail sanction on firsts time drunk drivers: a quasi experimental study. Accident, Analysis and Prevention, 25, 561-568.

McGuire, F.L. (1982). Treatment of the drinking driver. Health Psychology, 1, 137-152.
National Transportation Safety Board (NTBS) (1984). Safety Study: deficiencies in enforcement, judicial and treatment programs related to repeat offender drunk driver, NTSB/SS-84. Washington, D.C.: NTBS.

Nichols, J.L. y Ross, H.L. (1990). The effectiveness of legal sanctions in dealing with drinking drivers. Alcohol, Drugs and Driving, 6, 33-60.

Nichols, J.L., Weinstein, E.B., Ellingstad, V.S. y StruckmanJohnson, D.L. (1978). The specific deterrent effect of ASAP education and rehabilitation programmes. Journal of Safety Research, 10, 177-187.

Observatorio Español sobre Drogas (2002). Informe $n^{\circ} 5$. Madrid: Plan Nacional sobre Drogas.

Peck, R.C. (1991). The general and specific deterrent effect of DUI sanctions: A review of California's experience. Alcohol, Drugs and Driving, 7, 13-42.

Presidential Commission on Drunk Driving (1983). Final Report. Washington D.C.: GPO.

Preusser, D.F., Blomberg, R.D. y Ulmer, R.G. (1988). Evaluation of the 1982 Wisconsin drinking and driving law. Journal of Safety Research, 19, 29-40.

Reed, D.S. (1982). Dealing with the Drinking Driving problem. Alcohol Health and Research World, 7, 4-7.

Reis, R.E. (1980). The findings of the comprehensive driving under the influence of alcohol offender treatment demonstration project. Abstracts and Reviews in Alcohol and Driving, 4, 10-16.

Ross, H.L. (1975). The Scandinavian myth: the effectiveness of drinking and driving legislation in Sweden and Norway. Journal of legal studies, 4, 285-310.

Sadler, D.D. y Perrine, M.W. (1984). An evaluation of the California drunk driving countermeasure system: Volume 2. The long-term traffic safety impact of a pilot alcohol abuse treatment as an alternative to license suspensions. Sacramento, CA: Department of Motor Vehicles.

Sánchez, J.M. (1995). Peculiaridades acerca del comportamiento humano en tráfico. Intervención psicosocial, 11, 26-36.

Seixas, F.A. y Hopson, A.L. (1975). The effect of rehabilitation on the driving behavior of problems drinkers. En: S. Israelstam y S. Lambert (Eds.), Alcohol, Drugs and traffic safety. Proceedings of the 6th International Conference on Alcohol, Drugs and Traffic safety 723-736. Toronto: Addiction Research Foundation of Ontario.

Spoerer, E., Ruby, M.M.; y Jennsch, M. (1997). Back to the wheel. Cologne: Rot-Gelb-Grün Braunschweig.

Tábula V (1992). Actitudes sociales frente al riesgo vial en la conducción de vehículos SARTRE (encuesta de tráfico panaeuropea). [Informe interno].

Torres, M.A. y Cortés, M.T. (1998). Proyecto del Programme for Alcohol Impaired Drivers (PAID). En: J.A. García y C. López. (Eds.), Nuevas Aportaciones a la prevención de las Drogodependencias 239-261. Madrid: Síntesis.

Transportation Research Board/National Research Council (1995). Strategies for dealing with the persistent drinking driver, number 437. Washington: Transportation Research Circular.

Vingilis, E., Chung, E. y Adlaf, L. (1981). An evaluation of a prevention programme for drinking driving called R.I.D.E. En: L. Golberg (Ed.), Alcohol, Drugs and Traffic 
Safety. (vol. 3). Sweden: Almquist and Wiskell International.

Voas, R.B., Scott, E. y Lange, S. (1997). Evaluation of a method for reduced unlicense drinving: the Washingron and Oregon license plate stiker laws. Accident, Analysis and Prevention, 29, 627-634.

Wells-Parker, E. (1995). Mandated treatment: Lessons from research with drinking and driving offenders. Alcohol Health and Research World, 18, 302-306.

Wells-Parker, E. y Cosby, P.J. (1988). Behavioral and employment consequences of driver's license suspension for drinking driving offenders. Journal of Safety Research, 19, 5-20.

Winkler, W., Jacobshaguen, W. y Nickel, W. (1990). Long term effects of courses for repeatedly alcoholconspicuous drivers. Analysis after 60 months of probation. Blutalkohol, 27, 154-174.

Yu, J. y Williford, R.W. (1995). Problem drinking and high-risk driving: an analysis of official and self-reported drinkingdriving in New York State. Addiction, 88, 219, 